\title{
Signatures of quantum behavior in single-qubit weak measurements
}

\author{
Rusko Ruskov ${ }^{1 *}$, Alexander N. Korotkov ${ }^{2}$, and Ari Mizel ${ }^{1}$ \\ ${ }^{1}$ Department of Physics and Materials Research Institute, \\ Penn State University, University Park, Pennsylvania 16802, U.S.A. and \\ ${ }^{2}$ Department of Electrical Engineering, University of California, Riverside, CA 92521-0204, U.S.A.
}

(Dated: November 5, 2018)

\begin{abstract}
With the recent surge of interest in quantum computation, it has become very important to develop clear experimental tests for "quantum behavior" in a system. This issue has been addressed in the past in the form of the inequalities due to Bell and those due to Leggett and Garg. These inequalities concern the results of ideal projective measurements, however, which are experimentally difficult to perform in many proposed qubit designs, especially in many solid state qubit systems. Here, we show that weak continuous measurements, which are often practical to implement experimentally, can yield particularly clear signatures of quantum coherence, both in the measured correlation functions and in the measured power spectrum.
\end{abstract}

PACS numbers: 03.65.Ta, 03.65.Ud, 03.67.-a

Since the inception of quantum mechanics, physicists have tried to formulate a concise statement of its essential difference from classical mechanics [1, 2]. In the past, this has been an important endeavor mainly because it has facilitated the everyday use of quantum mechanics: by developing intuition about the non-classical aspects of the theory, one is better able to apply it to explain data. More recently, a remarkable new benefit has emerged from the effort. It turns out that some of the most subtle non-classical features of quantum mechanics actually have promising applications potential. Researchers have proposed quantum computers and other quantum information devices that could rely on quantum mechanical entanglement effects to qualitatively outperform their classical counterparts in some important tasks (see Refs. 3, 4).

Concerted effort is now being directed toward the fabrication and control of quantum systems that could constitute the components of a quantum information device. In particular, various two-state systems are being studied to see if they can be made to serve as "qubits" [5], the quantum computation analogue of the classical bit. An important practical issue arises naturally in such research. One would like to be able to verify that a given candidate system is capable of exhibiting rudimentary quantum behavior before attempting to construct an elaborate apparatus that can execute some task. How can an experimentalist demonstrate that a given system is being "quantum" rather than "classical"? Sometimes, researchers present oscillatory data and claim that their system is undergoing quantum Rabi oscillations. While generally the most likely source of the behavior is quantum mechanical oscillations, an alternate, classical explanation of the oscillations is also generally possible.

* On leave of absence from Institute for Nuclear Research and $\mathrm{Nu}-$ clear Energy, Sofia BG-1784, Bulgaria
In a profound and well-known paper [6], J.S. Bell formulated inequalities that must be obeyed by any local hidden variables theory; a system that violates the inequalities is necessarily exhibiting non-classical behavior. Unfortunately, the practical requirements involved in a test of Bell's theorem can be demanding. The system being tested must have two degrees of freedom that can be entangled, spatially separated, and then separately measured. These requirements are often too stringent to permit initial assessments of the potential of a candidate system to serve as a qubit.

In a different context, while seeking ways to test the predictions of quantum mechanics for macroscopic variables, Leggett and Garg have provided "Bell inequalities in time" [7]. These inequalities are designed for testing a system with just one degree of freedom and therefore can be much easier to apply in the laboratory than Bell's original inequalities. However, they still assume the ability to perform projective measurements on the system. In many systems that are currently under consideration as candidate components for quantum computers, repeated projective measurements are difficult or impossible to perform. Especially in solid-state systems such as superconducting Josephson junction qubits [8] one often performs only "weak" continuous measurements that probe the system gradually and indicate its state after accumulating enough information.

In this paper, we show how the "Bell inequalities in time" can be formulated to test a system that is probed with weak continuous measurements $9,10,11,12]$ rather than projective measurements. We show certain advantages to using weak rather than projective measurements (which also proved useful in quantum optics [13]). We provide an analysis of the weak measurement signal of a two-state system, pointing out constraints that hold (under appropriate conditions) for a classical but not for a quantum mechanical two-state system. When experimental data violate these constraints one therefore has a 
distinct signature of quantum behavior.

To formulate weak-measurement "Bell inequalities in time" consider a system with a physical characteristic described by the variable $Q(t)$. Assume that the system conforms with the following two axioms of macrorealism 7]: (A1) $Q(t)$ has a well-defined value at all times and (A2) it is possible to obtain the value of $Q(t)$ with a non-invasive measurement. Assume further that $Q(t)$ is bounded above and below so that, without loss of generality, we can arrange definitions so that $|Q(t)| \leq 1$.

Choose two non-vanishing time intervals $\tau_{1}$ and $\tau_{2}$. Then for any initial time $t$, by axiom (A1) the three numbers $Q(t), Q\left(t+\tau_{1}\right)$, and $Q\left(t+\tau_{1}+\tau_{2}\right)$ give characteristics of the system at times $t, t+\tau_{1}$, and $t+\tau_{1}+\tau_{2}$. They satisfy the inequality

$$
\begin{aligned}
Q(t) Q\left(t+\tau_{1}\right)+ & Q\left(t+\tau_{1}\right) Q\left(t+\tau_{1}+\tau_{2}\right) \\
& -Q(t) Q\left(t+\tau_{1}+\tau_{2}\right) \leq 1 .
\end{aligned}
$$

This is proved simply by maximizing the left hand side subject to the constraints $|Q(t)|,\left|Q\left(t+\tau_{1}\right)\right|, \mid Q\left(t+\tau_{1}+\right.$ $\left.\tau_{2}\right) \mid \leq 1$ [14.

When making weak measurements of the system, instead of obtaining simply $Q(t)$, one collects a noisy signal

$$
I(t)=I_{0}+\frac{\Delta I}{2} Q(t)+\xi(t)
$$

where $\xi(t)$ represents white noise [15] with vanishing time average $\langle\xi(t)\rangle \equiv \lim _{T \rightarrow \infty} \frac{1}{T} \int_{-T / 2}^{T / 2} \xi(t) d t=0$ and with $\delta$ function correlator $\langle\xi(t) \xi(t+\tau)\rangle=\frac{S_{0}}{2} \delta(\tau) ; S_{0}=2 e I_{0}$ is the spectral density and $-e$ is the electron charge. The symbol $I(t)$ is appropriate since the measured signal could be the current through a device like a quantum point contact [16, 17] (although our analysis is not limited to this case). The background signal is $I_{0}$ and $\Delta I$ is the difference between the signal associated with $Q(t)=1$ and $Q(t)=-1$. By appropriately averaging $I(t)$ to minimize noise, one can obtain information about $Q(t)$. In particular, for $\tau>0$ the time-averaged current correlation is

$$
\begin{aligned}
& K_{I}(\tau) \equiv\left\langle\left(I(t)-I_{0}\right)\left(I(t+\tau)-I_{0}\right)\right\rangle \\
& =\left(\frac{\Delta I}{2}\right)^{2}\langle Q(t) Q(t+\tau)\rangle+\frac{\Delta I}{2}\langle\xi(t) Q(t+\tau)\rangle .
\end{aligned}
$$

We have used equation (2) and the fact that $\langle Q(t) \xi(t+\tau)\rangle=0$, for a classical or a quantum system, as long as the state of the system does not anticipate the future random noise in the detector. Since it is possible to make measurements without disturbing the system (axiom (A2)), there is no reason that any correlation has to arise between the noise that registers in the detector and the physical characteristic $Q(t)$ of the system being measured. In particular, axiom (A2) implies that in principle one can arrange that

$$
\langle\xi(t) Q(t+\tau)\rangle=0
$$

Indeed, even experimentally plausible detector designs exist [18] that use "ideal negative-result" measurements 7] to minimize classical back-action of the detector on the system. Moreover, we have considered a model of weak continuous measurement in which detector noise linearly perturbs one of the energy parameters in the qubit Hamiltonian; despite the back-action explicitly included in this reasonable model, the back-action correlator in (41) still vanishes assuming "good symmetric oscillations", $\langle Q(t)\rangle=0$.

Using Eq. (4) we get $K_{I}(\tau)=(\Delta I / 2)^{2}\langle Q(t) Q(t+\tau)\rangle$. Averaging the inequality (11) over time $t$, we conclude

$$
K_{I}\left(\tau_{1}\right)+K_{I}\left(\tau_{2}\right)-K_{I}\left(\tau_{1}+\tau_{2}\right) \leq\left(\frac{\Delta I}{2}\right)^{2} .
$$

This is a "Bell inequality in time" for weak measurements. We will show momentarily that it is violated by a quantum system. Note that, aside from being convenient for application to realistic experiments, this form of the inequality has a compelling advantage over the projective measurement version. In the projective measurement version [7], one takes an ensemble average rather than a time average of the inequality (11) in order to arrive at an inequality that is violated in the quantum case. In addition, one introduces ensembles: one ensemble which is measured at times $t, t+\tau_{1}$ to provide the ensemble average of $Q(t) Q\left(t+\tau_{1}\right)$, one ensemble measured at times $t+\tau_{1}, t+\tau_{1}+\tau_{2}$ to provide the ensemble average of $Q\left(t+\tau_{1}\right) Q\left(t+\tau_{1}+\tau_{2}\right)$, and one ensemble measured at times $t, t+\tau_{1}+\tau_{2}$ (but definitely not at time $t+\tau_{1}$ ) to provide the ensemble average of $Q(t) Q\left(t+\tau_{1}+\tau_{2}\right)$. Only by refraining from measuring at time $t+\tau_{1}$ can one preserve the interference effects in a quantum system that alter the value of $Q(t) Q\left(t+\tau_{1}+\tau_{2}\right)$ and bring about a violation of the inequality. Because ensembles play such an important role, an additional explicit axiom of macrorealism called "induction" was introduced in Refs. 7] to stipulate that all ensembles have identical properties.

In the case of weak continuous measurements, no ensembles need to be introduced since a quantum system subjected to sufficiently weak measurements still can preserve quantum coherence [12]; the correlators appearing in (5) all refer to time averages of measurements performed continuously on a single system. (Of course, we have not attempted to uproot all unstated assumptions of "induction" from our analysis; inevitably there are many painfully "obvious" unstated axioms of this sort underlying any derivation. Our point is just that we have avoided the somewhat awkward problem of preparing ensembles of systems in the same starting state.)

We now demonstrate that a quantum mechanical twostate system undergoing weak measurements violates (5) under appropriate conditions. Consider the density matrix $\rho$ of the system with basis chosen so that the quantity $Q(t)=\rho_{11}-\rho_{22}$. Measurements and Hamiltonian evolution both produce changes in $\rho$. The following stochastic 
equations (in Stratonovich form) can be derived using informational (Bayesian) analysis or by treating the measurement device as a quantum system with finite coupling to the system being probed and then performing sufficiently frequent projective measurements on the measurement device [12]

$$
\begin{aligned}
& \dot{\rho}_{i j}=\rho_{i j} \frac{1}{S_{0}} \sum_{k} \rho_{k k}\left[\left(I(t)-\frac{I_{k}+I_{i}}{2}\right)\left(I_{i}-I_{k}\right)\right. \\
& \left.+\left(I(t)-\frac{I_{k}+I_{j}}{2}\right)\left(I_{j}-I_{k}\right)\right]-\gamma_{i j} \rho_{i j}-\frac{i}{\hbar}[\mathcal{H}, \rho]_{i j} .
\end{aligned}
$$

Here, $\mathcal{H}$ is the system Hamiltonian and $I(t)$ is the measurement result (2). The system decoherence rate is $\gamma_{i j}=(1 / \eta-1) \frac{\left(I_{i}-I_{j}\right)^{2}}{4 S_{0}}$, where the ideality $\eta$ is unity for an ideal detector like a quantum point contact 12, 19]. The value $I_{k}$ is the current through the detector when the system is in state $|k\rangle$. For our one-qubit case of interest, $k=1,2$ and $I_{1,2}=I_{0} \pm \Delta I / 2$. For simplicity, we take the Hamiltonian to have the form $\mathcal{H}=(\Omega / 2)(|1\rangle\langle 2|+| 2\rangle\langle 1|)$ and denote $\gamma_{12} \equiv \gamma$. Proceeding as in [20], stochastic equations imply

$$
\begin{gathered}
\langle Q(t) Q(t+\tau)\rangle= \\
\quad\left\langle Q^{2}(t)\right\rangle e^{-\Gamma \tau / 2}\left(\cos \tilde{\Omega} \tau+\frac{\Gamma}{2 \tilde{\Omega}} \sin \tilde{\Omega} \tau\right) \\
\quad\left\langle 2 \operatorname{Im} \rho_{12}(t) Q(t)\right\rangle e^{-\Gamma \tau / 2} \frac{\Omega}{\tilde{\Omega}} \sin \tilde{\Omega} \tau,(7) \\
\langle\xi(t) Q(t+\tau)\rangle \\
=\frac{\Delta I}{2}\left(1-\left\langle Q^{2}(t)\right\rangle\right) e^{-\Gamma \tau / 2}\left(\cos \tilde{\Omega} \tau+\frac{\Gamma}{2 \tilde{\Omega}} \sin \tilde{\Omega} \tau\right) \\
+\frac{\Delta I}{2}\left\langle 2 \operatorname{Im} \rho_{12}(t) Q(t)\right\rangle e^{-\Gamma \tau / 2} \frac{\Omega}{\tilde{\Omega}} \sin \tilde{\Omega} \tau
\end{gathered}
$$

where $\tilde{\Omega}=\sqrt{\Omega^{2}-\Gamma^{2} / 4}$ and the total decoherence rate is $\Gamma=\gamma+(\Delta I)^{2} / 4 S_{0}=(\Delta I)^{2} / 4 S_{0} \eta$. We conclude that the current correlation (3) in the quantum case has the form [20, 21]

$$
K_{I}(\tau)=\left(\frac{\Delta I}{2}\right)^{2} e^{-\Gamma \tau / 2}\left(\cos \tilde{\Omega} \tau+\frac{\Gamma}{2 \tilde{\Omega}} \sin \tilde{\Omega} \tau\right) .
$$

The second correlator, (8), shows an inevitable back action of noise from the detector into the evolution of the system. This "invasiveness" is an essential difference between a quantum system and a macrorealistic system satisfying axiom (A2) above. For an ideal $(\eta=1)$ detector and in the weak coupling regime $\Gamma \ll \Omega$, we find that $\left\langle Q^{2}(t)\right\rangle \rightarrow 1 / 2$ and $\left\langle 2 \operatorname{Im} \rho_{12}(t) Q(t)\right\rangle \sim \Gamma / \Omega$, so that the two correlators (7), (8) give an equal contribution to the total correlation function (9).

Choosing $\tau_{1}=\tau_{2}=\tau \ll 1 / \Gamma$ in the inequality (5) and using (9), we find in the weak coupling limit, $\Gamma \ll \Omega$, that

$$
\begin{aligned}
& K_{I}(\tau)+K_{I}(\tau)-K_{I}(2 \tau) \\
& =\left(\frac{\Delta I}{2}\right)^{2}\left(1+2\left(\cos \Omega \tau-\cos ^{2} \Omega \tau\right)\right) .
\end{aligned}
$$

This violates the inequality (5) provided that $0<$ $\cos (\Omega \tau)<1$. We get a maximum violation of (5) by choosing $\tau=\pi / 3 \Omega$; in this case the left hand side becomes $(3 / 2)(\Delta I / 2)^{2}$. When experimental data violate inequality (5), it demonstrates that the sample is not a macrorealistic system being probed by non-invasive measurements. If an experimentalist struggles to make noninvasive measurements but finds that the data inevitably violate (5), this provides evidence that the system is behaving non-classically. Naturally, when the decoherence rate $\Gamma$ becomes large in (9), it is no longer possible [22] to violate the inequality (5).

Often, instead of directly considering the correlator (3), it is experimentally convenient to analyze its power spectrum $S_{I}(\omega) \equiv 2 \int_{-\infty}^{\infty} d \tau K_{I}(\tau) e^{i \omega \tau}$. We now derive inequalities that constrain the area under peaks in the power spectrum. The presence of large area peaks that violate these inequalities should be regarded as evidence that the sample is not a macrorealistic system being probed non-invasively. We employ a lemma that relates the frequency filtration of the spectrum using a frequency window and time averaging of the current using a time window:

$$
\int_{-\infty}^{\infty}\left(S_{I}(\Omega+\omega)-S_{0}\right) f(\omega) \frac{d \omega}{2 \pi}=\frac{1}{\pi}\left\langle|J(\Omega, t)|^{2}\right\rangle,
$$

where $J(t) \equiv(\Delta I / 2) Q(t)$ is the "pure" signal. Here, $f(\omega)$ is a frequency window that goes to zero as $|\omega|$ increases. The Fourier transformed current signal is $J(\Omega, t)=\int_{-\infty}^{\infty} J(t+\tau) e^{i \Omega \tau} g(\tau) d \tau$ with time averaging over a time window $g(\tau)$; it is related to the frequency window as: $f(\omega)=\frac{1}{2 \pi} \int_{-\infty}^{\infty} g(\tau) g^{*}(t+\tau) e^{i \omega \tau} d \tau d t$. If one chooses a Gaussian window $f(\omega)=e^{-\omega^{2} / 2 \Delta^{2}}$ the lemma holds for a Gaussian time window $g(\tau)=\sqrt{2} \Delta e^{-\tau^{2} \Delta^{2}}$.

The integral (11) gives the area under a peak in the power spectrum centered at frequency $\Omega$ provided the width $\Delta$ of the frequency window is much larger than the peak width $W$. Assuming that the peak is sufficiently narrow, it is possible to have $W \ll \Delta \ll \Omega$. The right hand side of (11) involves a time average of $|J(\Omega, t)|^{2}$ that is bounded above by its maximum value $\max _{t}|J(\Omega, t)|^{2}$, attained at the time $t_{\max }$. Defining the phase $\phi$ by $J\left(\Omega, t_{\max }\right)=\left|J\left(\Omega, t_{\max }\right)\right| \exp (i \phi)$ we note that

$$
\begin{aligned}
& \left|J\left(\Omega, t_{\max }\right)\right|=\int_{-\infty}^{\infty} J\left(t_{\max }+\tau\right) e^{i(\Omega \tau-\phi)} g(\tau) d \tau \\
& \leq \int_{-\infty}^{\infty}\left|J\left(t_{\max }+\tau\right)\right||\cos (\Omega \tau-\phi)| g(\tau) d \tau \\
& \leq \int_{-\infty}^{\infty} \frac{\Delta I}{2}|\cos (\Omega \tau-\phi)| \sqrt{2} \Delta e^{-\Delta^{2} \tau^{2}} d \tau .
\end{aligned}
$$

In the above, both $t_{\max }$ and $\phi$ depend on the realization of the measurement process however the final estimate does not. In the final step, we note that $\Delta \ll \Omega$ implies $\int|\cos (\Omega \tau-\phi)| \exp \left(-\Delta^{2} \tau^{2}\right) d \tau=(2 / \sqrt{\pi} \Delta)[1+$ 
$\left.o\left(\frac{\Delta}{\Omega}\right)\right]$ since the average of the rapidly oscillating absolute value of cosine is $2 / \pi$. The correction term $o\left(\frac{\Delta}{\Omega}\right)$ rapidly decreases for small $\Delta$; it is less than $1 \%$ for $\frac{\Delta}{\Omega}<0.4$. One concludes that

$$
\int_{-\infty}^{\infty}\left(S_{I}(\Omega+\omega)-S_{0}\right) f(\omega) \frac{d \omega}{2 \pi}<\frac{8}{\pi^{2}}\left(\frac{\Delta I}{2}\right)^{2}\left[1+o\left(\frac{\Delta}{\Omega}\right)\right]
$$

is a bound on the area of any sufficiently narrow peak in the power spectrum of a macrorealistic system probed non-invasively. The upper limit of $8 / \pi^{2}(\Delta I / 2)^{2}$ cannot be improved without assuming further restrictions on the form of $I(t)$. To see this, note that the limit in (12) is actually attained by quasi-periodic rectangular oscillations: $Q(t)=Q_{R}(\Omega t+\varphi(t))$ where $Q_{R}(\theta)=1$ for $2 n \pi<\theta<$ $(2 n+1) \pi$ and $Q_{R}(\theta)=-1$ for $(2 n+1) \pi<\theta<2(n+1) \pi$, for $n=0,1, \ldots$ and where $\varphi(t)$ is a slowly fluctuating phase 25]. The bound (12) is violated by the quantum power spectrum [20, 21] obtained by Fourier transforming (9)

$$
S_{I}(\omega)=S_{0}+\left(\frac{\Delta I}{2}\right)^{2} \frac{4 \Omega^{2} \Gamma}{\left(\omega^{2}-\Omega^{2}\right)^{2}+\Gamma^{2} \omega^{2}}
$$

which has an area of $(\Delta I / 2)^{2}$ under the peak at frequency $\Omega$ [26]. If we assume that power spectrum displays only a single narrow peak, which is at non-zero frequency, then it is possible to reduce the bound (12). Consider a peak of functional form (13) generated by measurements of a classical system (in this case the peak width is $W$; it is not limited from below by $\left.(\Delta I)^{2} / 4 S_{0}\right)$. Suppose the prefactor of $(\Delta I / 2)^{2}$ is replaced with $(\Delta I / 2)^{2} K_{0}$ where $K_{0}$ is a constant factor. The Fourier transform of this power spectrum is a correlation function of the form (9) with prefactor $(\Delta I / 2)^{2}$ replaced by $(\Delta I / 2)^{2} K_{0}$. Assuming that this is the output of a classical system, $K_{0}$ is then constrained by (5). Taking $\tau_{1}=\tau_{2}=\tau=\pi / 3 \Omega$ and assuming $W \tau \ll 1$, we find that $K_{0} \leq 2 / 3$. Thus, in this case

$$
\int_{-\infty}^{\infty}\left(S_{I}(\Omega+\omega)-S_{0}\right) f(\omega) \frac{d \omega}{2 \pi} \leq \frac{2}{3}\left(\frac{\Delta I}{2}\right)^{2} .
$$

The assumption of a single narrow peak in $S_{I}(\omega)$ has led to a more stringent constraint on the relative peak area of $2 / 3$. One can find a classical process with a single Lorenzian peak of area 1/2 (e.g., $Q(t)=\cos (\Omega t+\varphi(t))$ with slowly varying phase $\varphi(t)$.) Thus the exact upper bound in the case of a single Lorenzian peak is between $1 / 2$ and $2 / 3$.

The three constraints (5), (12), and (14) provide powerful and convenient means of testing the nonclassicalness of a system. Rather than simply pointing to an oscillatory signal and claiming quantum coherent oscillations, an experimentalist can use these inequalities to demonstrate conclusively the violation of macroscopic, non-invasive behavior. While it is always possible that the experimentalist is inadvertently performing invasive measurements on a classical system, this possibility becomes increasingly unlikely as more effort is exerted to make the measurements non-invasive. Although we have focused here upon a single qubit, with little modification one can apply these constraints to weak measurements on systems with two or more degrees of freedom.

The authors gratefully acknowledge the support of the Packard foundation (R.R. and A.M.) and of NSA/ARDA/ARO (A.K.).

[1] N. Bohr, Phys. Rev. 48, 696 (1935).

[2] A. Einstein, B. Podolsky, and N. Rosen, Phys. Rev. 47, 777 (1935).

[3] C. H. Bennett, Physics Today 48 (10), 24 (1995).

[4] M. Nielsen and I. L. Chuang, Quantum computation and quantum information, Cambridge University Press, Cambridge, 2000.

[5] For example, D. Loss and D. P. DiVincenzo, Phys. Rev. A 57, 120 (1998); A. Shnirman, G. Schön, and Z. Hermon, Phys. Rev. Lett. 79, 2371 (1997). D. V. Averin, Solid State Commun. 105, 659 (1998); B. E. Kane, Nature 393, 133 (1998); J. E. Mooij et al., Science 285, 1036 (1999).

[6] J. S. Bell, Physics (Long Island City, N.Y.), 1, 195 (1964).

[7] A. J. Leggett and A. Garg, Phys. Rev. Lett. 54857 (1985); A. J. Leggett, J. Phys.: Condens. Matter 14, R415 (2002).

[8] Y. Nakamura, Yu. A. Pashkin, and J. S. Tsai, Nature (London) 398, 786 (1999); C. H. van der Wal, A.C.J. ter Haar, F. K. Wilhelm, R. N. Schouten, C.J.P.M. Harmans, T. P. Orlando, S. Lloyd, and J. E. Mooij, Science 290, 773 (2000); J. R. Friedman, V. Patel, W. Chen, S. K. Tolpygo, and J. E. Lukens, Nature (London) 406, 43 (2000); D. Vion, A. Aassime, A. Cottet, P. Joyez, H. Pothier, C. Urbina, D. Esteve, M. H. Devoret, Science 296, 886 (2002); J. M. Martinis, S. Nam, J. Aumentado, C. Urbina, Phys. Rev. Lett. 89, 117901 (2002).

[9] M. B. Mensky, Physics-Uspekhi 168, 1017 (1998).

[10] H. J. Carmichael, An open system approach to quantum optics, Lecture notes in physics (Springer, Berlin, 1993).

[11] H. M. Wiseman and G. J. Milburn, Phys. Rev. Lett. 70, 548 (1993).

[12] A. N. Korotkov, Phys. Rev. B 60, 5737 (1999); Phys. Rev. B 63, 115403 (2001).

[13] Physically distinct classical inequalities have been derived in quantum optics. See, e.g., R. Loudon, Rep. Prog. Phys., 43913 (1980) and H.J. Carmichael, H.M. CastroBeltran, G.T. Foster, and L.A. Orozco, Phys. Rev. Lett. 85, 1855 (2000).

[14] This inequality is identical to the inequality of 7] with the simple redefinition $Q\left(t+\tau_{1}\right) \rightarrow-Q\left(t+\tau_{1}\right)$; here we note that it applies not only under the assumption $Q= \pm 1$ but also under the assumption $|Q| \leq 1$.

[15] C. W. Gardiner, Handbook of Stochastic methods (Springer, Berlin, 1983).

[16] S. A. Gurvitz, Phys. Rev. B 56, 15215 (1997).

[17] E. Buks, R. Schuster, M. Heiblum, D. Mahalu, and V. Umansky, Nature 391, 871 (1998). 
[18] C. D. Tesche, Phys. Rev. Lett. 642358 (1990).

[19] S. Pilgram and M. Büttiker, Phys. Rev. Lett. 89, 200401 (2002); A. A. Clerk, S. M. Girvin, A. D. Stone, Phys. Rev. B 67, 165324 (2003).

[20] A. N. Korotkov and D. V. Averin, Phys. Rev. B 64, 165310 (2001); A. N. Korotkov, Phys. Rev. B 63, 085312 (2001).

[21] H.-S. Goan and G. J. Milburn, Phys. Rev. B 64, 235307 (2001); R. Ruskov and A. N. Korotkov, Phys. Rev. B 67, 075303 (2003); A. Shnirman, D. Mozyrsky, and I. Martin, Europhys. Lett., 67 (5), 840 (2004); L. N. Bulaevskii and G. Ortiz, Phys. Rev. Lett. 90, 040401 (2003).

[22] In particular, in the strong coupling limit, $\Gamma \gg \Omega(\eta$ fixed), the quantum Zeno effect 23] develops and corre- lator (9) corresponds to classical telegraph noise 20, 24]; then $\left\langle Q^{2}(t)\right\rangle=1$ and the quantum correlation (8) vanishes.

[23] B. Misra and E. C. G. Sudarshan, J. Math. Phys. 18, 756 (1977).

[24] Yu. Makhlin, G. Schön, A. Shnirman, Phys. Rev. Lett. 85, 4578 (2000).

[25] This random process exhibits narrow peaks at $\Omega_{n}=$ $(2 n+1) \Omega$ with area $8 /\left[\pi^{2}(2 n+1)^{2}\right](\Delta I / 2)^{2}$.

[26] It does not depend on $\Gamma$ and quantum efficiency $\eta$. However, the measurement time $\tau_{m}$ to accumulate the spectrum does increase for decreasing $\eta$; in particular, $\tau_{m} \gtrsim 8 S_{0} /(\Delta I)^{2} \eta$ for small $\eta$. 